V. Seidel

Starthilfe

Elektrotechnik 


\section{Starthilfe Elektrotechnik}

Von Prof. Dr.-Ing. habil. Volkmar Seidel FH Merseburg

B. G. Teubner Stuttgart · Leipzig · Wiesbaden 2000 
Prof. Dr.-Ing. habil. Volkmar Seidel

Geboren 1940 in Leipzig. Ab 1958 Studium der Elektrotechnik an der TU Dresden. Diplom 1964. Promotion 1974 an der Hochschule für Verkehrswesen „Friedrich List" Dresden. Von 1964 bis 1989 Industrietätigkeit im VEB Fernmeldewerk/Nachrichtenelektronik Leipzig als Entwicklungsingenieur und Themenleiter auf den Gebieten der Schaltungsentwicklung, Mikrorechentechnik und Softwareentwicklung. 1984 Habilitation in Dresden. 1989 Hochschuldozent für. Elektrotechnik an der TH Merseburg. Von 1993 bis 1996 Gründungsdekan des Fachbereichs Elektrotechnik und seit 1993 Professor für Grundlagen der Elektrotechnik an der FH Merseburg.

Die Deutsche Bibliothek - CIP-Einheitsaufnahme

Ein Titeldatensatz für diese Publikation ist bei

Der Deutschen Bibliothek erhältlich.

Das Werk einschließlich aller seiner Teile ist urheberrechtlich geschützt. Jede Verwertung außerhalb der engen Grenzen des Urheberrechtsgesetzes ist ohne Zustimmung des Verlages unzulässig und strafbar. Das gilt besonders für Vervielfältigungen, Übersetzungen, Mikroverfilmungen und die Einspeicherung und Verarbeitung in elektronischen Systemen. (C) B.G.Teubner Stuttgart · Leipzig · Wiesbaden 2000

Der Verlag Teubner ist ein Unternehmen der Fachverlagsgruppe BertelsmannSpringer.

Konzeption und Layout des Umschlags: Peter Pfitz, Stuttgart

ISBN-13:978-3-519-00264-2

e-ISBN-13:978-3-322-80016-9

DOI: 10.1007/978-3-322-80016-9 


\section{Vorwort}

Das vorliegende Buch richtet sich vorrangig an Schüler und Studienanfänger, die das Studium einer technischen Fachrichtung, insbesondere das Studium der Elektrotechnik, aufnehmen wollen.

Mit der Starthilfe möchte der Autor den Übergang von der Schule zur Hochschule und damit den Einstieg in ein rationelles Studium erleichtern. Sie knüpft an die Elektrizitätslehre des Physikunterrichts der Gymnasien an und vertieft und erweitert die dort erworbenen Kenntnisse.

Die Elektrotechnik ist ein weitgespanntes Gebiet und gliedert sich grob in Energietechnik und Informationstechnik. Die Energietechnik oder Leistungselektrik befaßt sich mit der Erzeugung und Verteilung elektrischer Energie. Die Geräte und Maschinen weisen daher infolge großer Leiterquerschnitte und beachtlicher Verlustwärme große Abmessungen auf. Gegenstand der Informationstechnik ist die Übertragung und Wandlung von Signalen. Hier dominieren mikroelektronische Schaltungen.

Der Studiengang Elektrotechnik besteht aus Grundstudium und Hauptstudium. Während das Grundstudium für alle Studenten gleich ist, können im Hauptstudium viele unterschiedliche Studienrichtungen gewählt werden. Der Zugang zu den Spezialrichtungen wird in dem Fach Grundlagen der Elektrotechnik im Grundstudium vorbereitet.

Pädagogisch besonders bewährt hat sich die Einteilung dieses Faches in die vier Abschnitte Gleichstrom, Elektrisches Feld, Elektromagnetismus und Wechselstrom. Aus dieser Einteilung werden die Unterschiede zur Elektrizitätslehre der Physik deutlich. Diese beginnt mit der Elektrostatik und erläutert den Ursache-Wirkungs-Zusammenhang, während die Technik den Zweck-Mittel-Zusammenhang betont. Im Abschnitt Gleichstromtechnik werden die elektrischen Größen Strom, Spannung und Widerstand definiert und die Kirchhoffschen Sätze behandelt und angewendet. Das typische Schaltelement ist der Ohmsche Widerstand $R$. Die Kirchhoffschen Sätze ermöglichen die Analyse elektrischer Schaltungen durch Berechnung der Ströme bei Vorgabe der Quellspannungen. Eine besondere Bedeutung haben die physikalischen Größen Leistung und Energie in der Elektrotechnik. Elektrische Energie läßt sich schnell und verlustarm transportieren. Sie wird durch Wandlung aus anderen Energieformen gewonnen und kann ebenso verlustarm wieder in andere Energieformen zurückgewandelt werden. Den Umwandlungsvorgängen wird besondere Aufmerksamkeit geschenkt.

Im Gegensatz zum linienhaften elektrischen Leiter nehmen schwach leitende Stoffe und Isolatoren Räume in Anspruch. Räumlich verteilte elektrische Größen, wie die Stromdichte und die elektrische Feldstärke, führen auf den Begriff des elektrischen Feldes. Der Kondensator, gekennzeichnet durch seine Kapazität $C$, ist hier das typische Schaltelement.

Die enge Verkopplung von elektrischen und magnetischen Größen wird im Abschnitt Elektromagnetismus behandelt. Das Durchflutungsgesetz und das Induktionsgesetz werden in ihren mathematischen Formulierungen als Maxwellsche Gleichungen bezeichnet und sind sowohl die Grundlage der großtechnischen Erzeugung von Elektroenergie als auch des Auftretens und der Ausbreitung von elektromagnetischen Wellen. Die Spule mit ihrer Induktivität $L$ tritt hier als typisches Schaltelement auf.

Der letzte Abschnitt führt in das Gebiet der Wechselströme ein. Sinuszeitfunktionen werden durch zwei Größen beschrieben, die Amplitude und den Winkel. Daher haben Vektoren in der Ebene, die als Zeiger bezeichnet werden, zentrale Bedeutung. Wechselströme und -span- 
nungen werden graphisch als Zeiger und mathematisch als komplexe Zahlen dargestellt. Aufgaben zur Addition von sinusförmigen Wechselgrößen werden von den Studierenden häufig falsch gelöst. Deshalb wird dieses Thema ausführlich behandelt. Ausgehend vom Additionstheorem der Kosinusfunktion führt der Weg über die anschauliche Zeigeraddition mit dem Kosinussatz für Dreiecke zur Lösung der Aufgabe mit komplexen Größen. Für die Wechselstromtechnik ist die Rechnung mit komplexen Größen elementar und unverzichtbar.

Wechselstromwiderstände haben zwei Komponenten, den Wirk- und den Blindwiderstand, oder in Polarkoordinaten, den Scheinwiderstand und den Winkel, und können daher ebenfalls durch komplexe Größen ausgedrückt werden.

Hauptschwierigkeit im ersten Semester ist die Anwendung der Mathematik auf technisch-naturwissenschaftliche Aufgabenstellungen. Im Abschnitt Gleichstrom treten bei der Analyse von Schaltungen Gleichungssysteme auf, deren Lösung in das Gebiet der Linearen Algebra fällt. In den Abschnitten Elektrisches Feld und Elektromagnetismus werden vektorielle Feldgrößen verwendet. Neben der elementaren Vektoralgebra werden auch Integrale über Skalarprodukte von Vektoren, die Hüllen- und die Linienintegrale, eingeführt. Diese Gleichungen können beim ersten Lesen übersprungen werden. Formal ist ein solches Integral über ein Skalarprodukt die Auflösung der Gleichung für eine vektorielle Feldgröße, die durch Differentiation einer skalaren Größe nach einem Vektor definiert wurde.

Die Strom-Spannungs-Beziehungen an einer Kapazität oder Induktivität enthalten einen Differentialquotienten. Die Behandlung der Schaltvorgänge an Netzwerken mit einer Kapazität oder einer Induktivität erfordert bereits die Lösung einer einfachen Differentialgleichung erster Ordnung. Sie gelingt durch Integration ohne tieferes Eindringen in die Theorie. Der zeitliche Verlauf der Kondensatorspannung kann durch eine Exponentialfunktion beschrieben werden. Damit wird die rein statische Betrachtungsweise elektrischer Schaltungen verlassen.

Der Start in das Studium einer technischen Fachrichtung ist nicht leicht. Beim ersten Lesen dieses Buches bleiben daher sicher Fragen offen, die in ausführlicher Form Gegenstand der Lehrveranstaltungen der beiden ersten Semester sind. Das sollte nicht entmutigen, sondern eher dazu motivieren, die im Anhang zitierte Fachliteratur zu befragen.

Der Autor wünscht dem Leser Freude beim Erkenntnisgewinn und einen guten Start in das Studium.

An dieser Stelle möchte ich Herrn Dr. Fiedler und Herrn Heinisch für die Durchsicht des Manuskriptes danken. Meiner Frau danke ich für das Korrekturlesen. Einigen Studenten gilt ebenfalls mein Dank. Herr Münchow gab nützliche Hinweise zur Verbesserung der Verständlichkeit der Darstellung. Herr Peltsch half mir, die Tücken des Textverarbeitungssystems zu bewältigen.

Weiterhin danke ich Herrn J. Weiß vom Teubner-Verlag für die angenehme Zusammenarbeit und die gute Beratung. 


\section{Inhalt}

$1 \quad$ Gleichstromtechnik $\ldots \ldots \ldots \ldots \ldots \ldots \ldots \ldots \ldots \ldots$

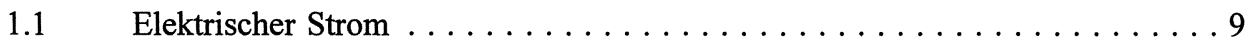

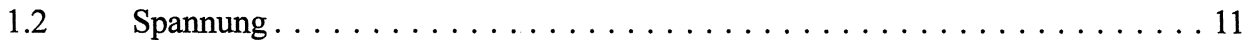

$1.3 \quad$ Widerstand . . . . . . . . . . . . . . . . . . . . . 13

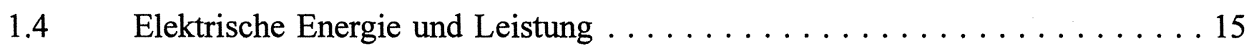

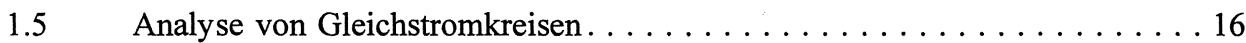

1.5.1 Kirchhoffsche Sätze. . . . . . . . . . . . . . . . . . . . . 16

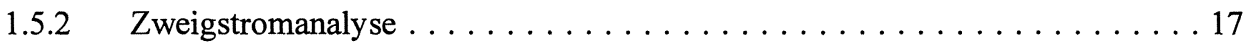

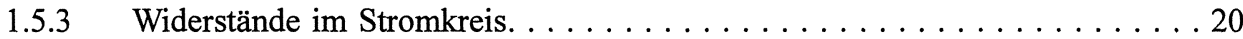

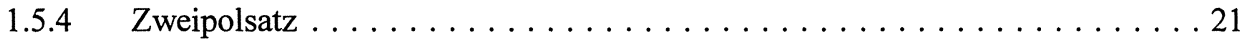

$1.6 \quad$ Leistung im Gleichstromkreis....................... 21

1.7 Wandlung elektrischer Energie in andere Energieformen $\ldots \ldots \ldots \ldots 23$

1.7.1 Wandlung elektrischer Energie in mechanische Energie . . . . . . . . . 23

1.7.2 Wandlung elektrischer Energie in Wärme. . . . . . . . . . . 24

1.7.3 Wandlung elektrischer Energie in chemische Energie. . . . . . . . . 26

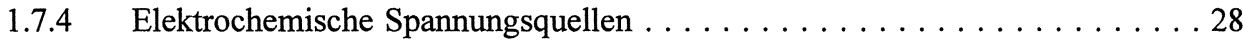

1.7.5 Wandlung von elektrischer Energie in Lichtenergie . . . . . . . . . . . . 29

1.7.6 Solarzelle, Wirkungsweise $\ldots \ldots \ldots \ldots \ldots \ldots \ldots \ldots \ldots \ldots \ldots \ldots \ldots \ldots \ldots$

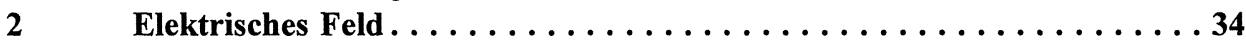

$2.1 \quad$ Flächenförmige und räumliche Leiter $\ldots \ldots \ldots \ldots \ldots \ldots \ldots \ldots \ldots$

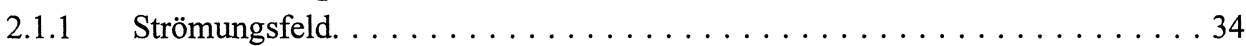

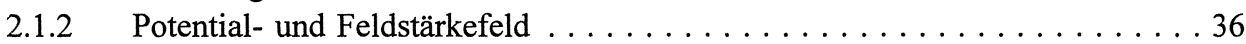

$2.2 \quad$ Elektrisches Feld im Nichtleiter . . . . . . . . . . . . . . . . 38

2.2.1 Verschiedene Feldformen . . . . . . . . . . . . . . . . . . . . . 38

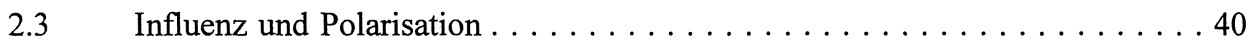

2.4 Dielektrischer Strom und Verschiebungsfluß .............. 41

$2.5 \quad$ Kondensator . . . . . . . . . . . . . . . . . . . . . . . . 42

$2.6 \quad$ Schaltungen mit Kondensatoren $\ldots \ldots \ldots \ldots \ldots \ldots \ldots \ldots \ldots \ldots$

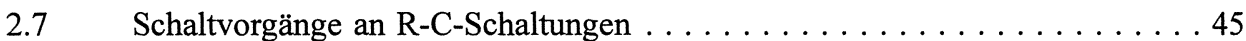

$2.8 \quad$ Kapazitäten von speziellen Kondensatoren und Leitungen . . . . . . . . . 47

$2.9 \quad$ Energie im elektrischen Feld $\ldots \ldots \ldots \ldots \ldots \ldots \ldots \ldots \ldots \ldots$

$2.10 \quad$ Kraft im elektrischen Feld $\ldots \ldots \ldots \ldots \ldots \ldots \ldots \ldots \ldots \ldots \ldots$

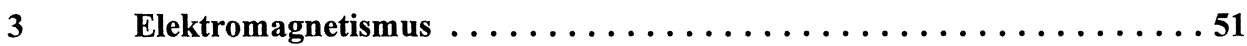

$3.1 \quad$ Magnetischer Kreis . . . . . . . . . . . . . . . . . . . 51

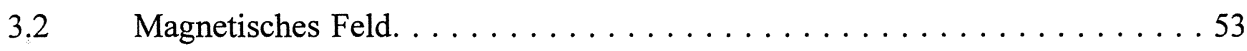

3.3 Magnetische Eigenschaften der Materie . . . . . . . . . . . . . 54

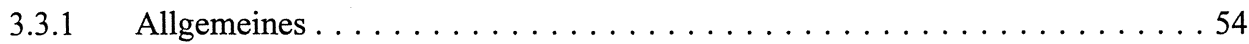

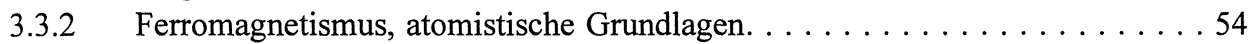

3.3.3 Magnetische Werkstoffe......................... 55

3.3.4 Feldverhalten an Trennflächen unterschiedlicher Permeabilität . . . . . . . 56 
$3.4 \quad$ Berechnung von magnetischen Kreisen $\ldots \ldots \ldots \ldots \ldots \ldots \ldots \ldots$

3.4.1 Erregung durch den elektrischen Strom . . . . . . . . . . . . . . . 56

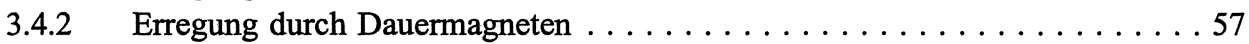

$3.5 \quad$ Kopplung elektrischer und magnetischer Größen. . . . . . . . . . . . 58

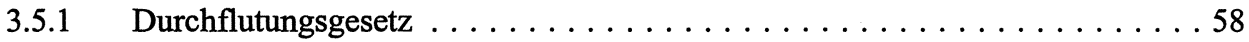

3.5.2 Anwendung des Durchflutungsgesetzes . . . . . . . . . . . . . . . 59

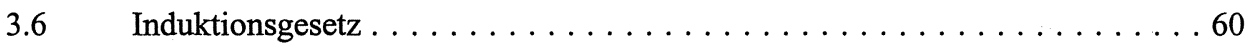

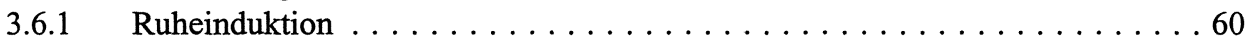

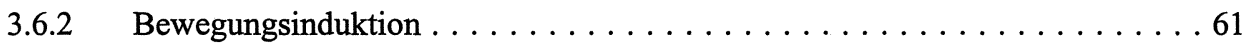

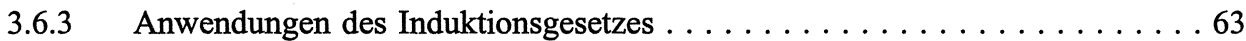

3.7 Wechselwirkung elektrischer und magnetischer Größen . . . . . . . . . . 64

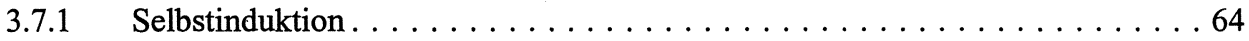

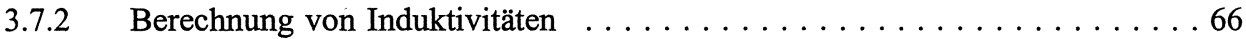

3.7 .3 Gegeninduktion. . . . . . . . . . . . . . . . . . . 67

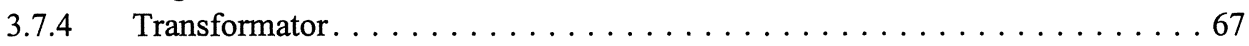

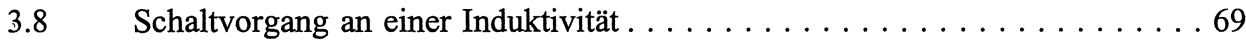

3.9 Energie im magnetischen Feld $\ldots \ldots \ldots \ldots \ldots \ldots \ldots \ldots \ldots \ldots$

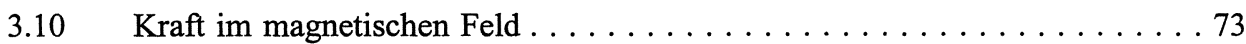

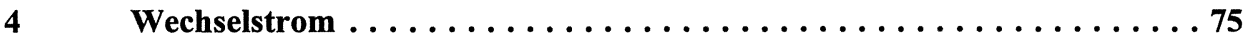

4.1 Begriff und Bedeutung des Wechselstromes . . . . . . . . . . 75

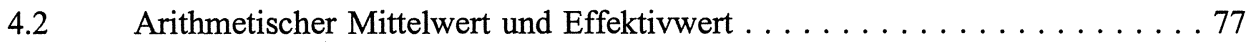

4.3 Lineare Operationen mit Sinusgrößen . . . . . . . . . . . . . 80

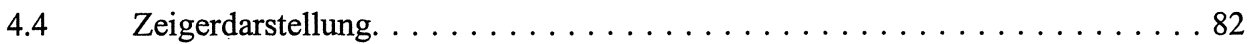

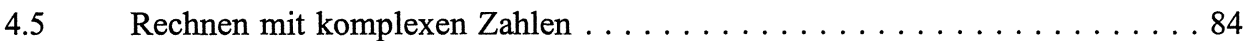

4.6 Symbolische Methode der Wechselstromtechnik . . . . . . . . . . 86

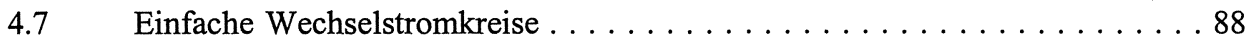

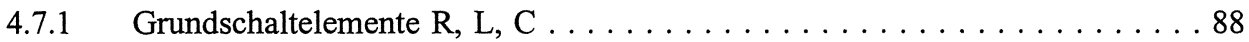

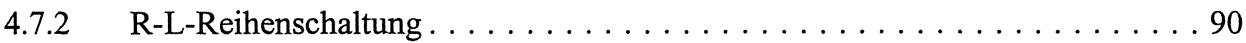

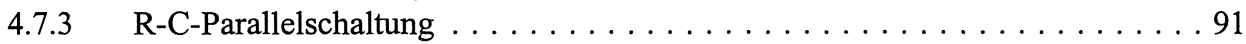

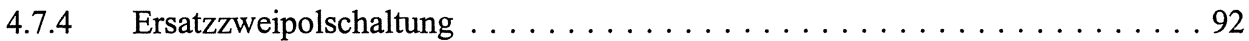

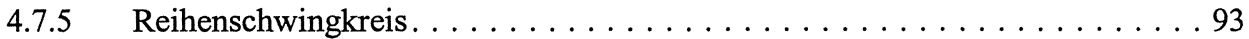

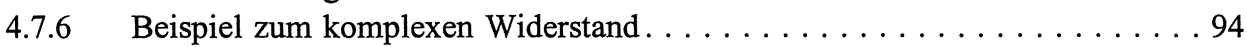

$4.7 .7 \quad$ Zeigerbilder . . . . . . . . . . . . . . . . . . . . . . . . . . . . . . . . . .

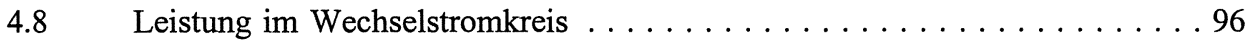

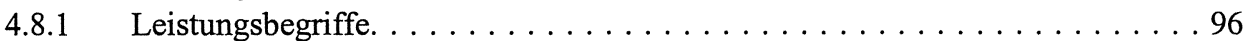

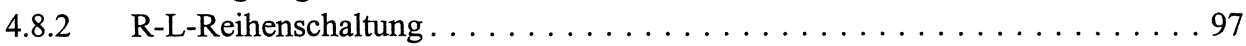

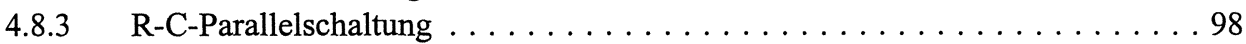

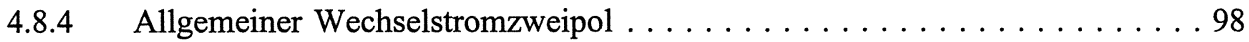

4.8.5 Zerlegung der Spannung und des Stromes in Wirk- und Blindkomponenten. . 99

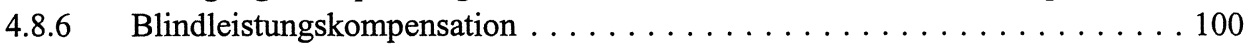

4.9 Beispiel zur Anwendung der komplexen Rechnung . . . . . . . . . 102

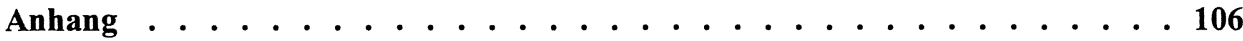

Stichwortverzeichnis ....................110 